\title{
Multiple reciprocity boundary face method for transient heat conduction in functionally graded materials
}

\author{
Shuaiping Guo, Guangyao Li \& Jianming Zhang \\ State Key Laboratory of Advanced Design and Manufacturing \\ for Vehicle Body, Hunan University, China
}

\begin{abstract}
Three dimensional functionally graded material is presented. It is assumed that the thermal conductivity varies exponentially along one direction. The heat generation and non-uniform initial distribution can be considered in the simulation. In the process of solving this diffusion problem numerically, the Laplace transform will be used to remove the time dependence of the problem. And the multiple reciprocity formulation of the modified Helmholtz fundamental solutions is used to convert the domain integral into boundary integrals. The results of test calculations are in agreement with exact solutions and finite element method simulations.

Keywords: transient heat conduction, functionally graded materials, boundary face method, Laplace transformation, multiple reciprocity method.
\end{abstract}

\section{Introduction}

Transient heat conduction problems can be efficiently solved by the boundary integral equation method [1]. The various solution procedures reported in the literature can essentially be classified into two broad categories: the time domain approach and the transform space approach [1-3]. The first boundary integral solution for the diffusion equation was proposed by Rizzo and Shippy [2]. In their approach, the time dependence of the problem is temporarily removed with the Laplace transform. Using the transform, the parabolic heat conduction equation is transformed into a more tractable elliptic equation. Then the boundary integral equation is derived and solved in the Laplace space for a sequence of real positive values of the transform parameter. At last, the inverse 
transformation is performed to evaluate the physical variables in the real space. Chang et al. [3] employed time-dependent fundamental solutions in the context of the direct method to solve two-dimensional problems of heat conduction in isotropic and anisotropic media in 1973. The both categories of approaches suffer from the time-consuming domain integral calculation.

In the unsteady heat conduction problem, the domain integral is necessary in the cases where the initial temperature distribution is not uniform and the heat generation function is arbitrary. In these cases, the basic advantage of dimensionality reduction is lost in the BEM. To avoid the domain integration, several methods have been proposed. Tanaka et al. [4] introduced the dual reciprocity method (DRM) to solve the diffusion problem. Nowak and Neves [5] applied the multiple reciprocity method (MRM) to convert the domain integral to an infinite series of boundary integrals using the higher-order fundamental solutions. In the MRM, however, numerical instability was found when the length of time step is small. Based on the MRM, Ochiai and Kitayama [6] proposed a triple reciprocity method. Gao [7] proposed the radial integration method (RIM) to evaluate the domain integrals with boundary-only discretization. In some cases, however, this method still applies internal points for variable interpolation.

The author applied the Laplace transformation technology to eliminate the time-dependence of the governing equation, and found the fundamental solution of the governing equation in LS had quickly convergent higher order fundamental solutions. So it is seemly to employ the MRM to convert the domain integrals, which are related to the non-union initial distribution and heat generations. The domain integrals will be converted to an infinite series of boundary integrals. And the quickly convergent higher order fundamental solutions make sure that the infinite series can also be computed through several truncated terms. The convergence had been discussed in Reference [8].

Functionally graded material (FGM) is a class of composites having continuous spatial variation of material properties. FGM is manufactured from isotropic components such as metals and ceramics, can resist severe thermal loading and maintain structural strength and toughness. In recent years, heat conduction problems in FGM have been investigated by several researchers. Sutradhar and Paulino [9] presented the Galerkin BEM with Laplace transform for heat conduction problem in homogeneous and non-homogeneous FGM. Sladek et al. [10] proposed a meshless method for transient heat conduction analysis in FGM using local boundary integral equation. Wang et al. [11] developed a virtual boundary collocation method (VBCM) based on RBF interpolation and time discretization to analyze this problem. Mirzaei and Dehghan [12] presented a new implementation of the meshless local BIE for this problem, in which the moving least squares (MLS) is used for approximating the temperature and heat flux in both time and space domains. But in these literatures the non-union initial condition and heat source are not considered.

In this paper, the heat source will be considered. Using the substitution represented in Reference [9], the governing equation will be a standard diffusion equation. And the heat generation function will become more complicated in the 
diffusion equation. The Laplace Transformation and Multiple Reciprocity Method (LTMRM) will be used to simulate the transient heat conduction in functionally graded materials. The domain integrals will be converted to the boundary integrals successfully.

After solving the BIE in LS, the Gaver-Wynn-rho algorithm is applied to transform the solution to the real space [13]. To avoid the geometric errors between the geometric model and the analysis model, our method is implemented in the framework of boundary face method (BFM) program [14, $15]$.

\section{Problem definition}

\subsection{Governing equation, boundary conditions and initial conditions}

The transient heat conductivity problem can be represented as:

$$
\nabla \cdot(k \nabla \phi)=c \rho \frac{\partial \phi}{\partial t}-h(Q, t) \quad(Q \in \Omega),
$$

where $\phi=\phi(Q, t)$ is the temperature of the location $Q$ at the time $t$. The coefficients $k, c$ and $\rho$ are the heat conductivity, the specific heat and the density, respectively. The function $h(Q, t)$ is the heat source density. $\Omega$ stands for the considered domain enclosed by $\Gamma_{1} \mathrm{U} \Gamma_{2}$. The boundary conditions are given as the following types:

$$
\begin{array}{ll}
\phi(Q, t)=\bar{\phi}(Q, t) . & x \in \Gamma_{1} \\
q(Q, t)=\frac{\partial \phi(Q, t)}{\partial n}=\bar{q}(Q, t) . & x \in \Gamma_{2},
\end{array},
$$

in which $\bar{u}, \bar{q}$ stand for the prescribed temperature and normal flux on the boundary respectively. Initial conditions at time $t=t_{0}$ can be prescribed as

$$
\phi_{0}(x)=\phi\left(x, t_{0}\right) . \quad(x \in \Omega)
$$

The thermal conductivity of the FGM is defined by an exponential function

$$
k(x, y, z)=k(z)=k_{0} e^{2 \beta z},
$$

in which $\beta$ is the material non-homogeneity parameter. The specific heat is also graded with the same functional variation as the conductivity,

$$
c(x, y, z)=c(z)=c_{0} e^{2 \beta z} .
$$

Substituting these material expressions into eqn. (1), one obtains

$$
\frac{\partial \phi}{\partial t}=\alpha\left(\nabla^{2} \phi+2 \beta \phi_{z}\right)+\frac{1}{c \rho} h(Q, t)
$$

where $\alpha=\mathrm{k}_{0} /\left(\mathrm{c}_{0} \rho\right)$. If one employs the substitution

$$
\phi=e^{-\beta z-\beta^{2} \alpha t} \times u,
$$

it will be obtained that 


$$
\frac{\partial u}{\partial t}=\alpha \nabla^{2} u+\frac{1}{c \rho} \tilde{h}(Q, t)
$$

which is the standard diffusion equation for a homogeneous material problem. We denote for short

$$
\tilde{h}(Q, t)=h(Q, t) \times e^{\beta z+\beta^{2} \alpha t} .
$$

The boundary conditions for eqn. (8) are

$$
\begin{array}{ll}
u(Q, t)=\bar{u}(Q, t)=\bar{\phi}(Q, t) \times e^{\beta z+\beta^{2} \alpha t} . & x \in \Gamma_{1} \\
q(Q, t)=\frac{\partial \bar{u}(Q, t)}{\partial n}=\bar{q}(Q, t) . & x \in \Gamma_{2}
\end{array}
$$

\subsection{MRM in Laplace space}

We denote the Laplace transform (LT) of a function $u(Q, t)$ by

$$
\tilde{u}(Q, s)=L(u(Q, t))=\int_{0}^{\infty} u(Q, t) \times e^{-s t} d t .
$$

And the heat source function after LT is

$$
\begin{aligned}
h(Q, s) & =\int_{0}^{\infty} h(x, t) \times e^{\beta z+\beta^{2} \alpha t} \times e^{-s t} d t \\
& =e^{\beta z} \int_{0}^{\infty} h(x, t) \times e^{-\left(s-\beta^{2} \alpha\right) t} d t=e^{\beta z} \times \tilde{h}\left(Q, s-\beta^{2} \alpha\right)
\end{aligned} .
$$

Implementing the LT on eqn. (8), we have the following governing equation:

$$
\nabla^{2} \tilde{u}-\lambda \tilde{u}=-\frac{1}{\alpha} u_{0}-\frac{1}{k_{0}} h(Q, s),
$$

where $\lambda=\mathrm{s} / \alpha$. It is worth noting that this is actually a modified Helmholtz equation. The fundamental solutions of the eqn. (13) are

$$
\begin{aligned}
& u^{*}=\frac{-1}{4 \pi r} e^{-\sqrt{\lambda} r} \\
& q^{*}=\frac{\partial u^{*}}{\partial n}=\frac{1}{4 \pi r^{2}}(1+\sqrt{\lambda} r) e^{-\sqrt{\lambda} r} \times \frac{\partial r}{\partial n},
\end{aligned}
$$

which satisfied the following equation:

$$
\nabla^{2} u^{*}-\lambda u^{*}=\Delta(Y, Q) \text {. }
$$

By employing the fundamental solutions in eqn. (14), eqn. (12) can be converted into the following BIE:

$$
\int_{\Gamma} q^{*} \cdot \tilde{u} d \Gamma-c \cdot \tilde{u}(Y)-\int_{\Gamma} u^{*} \cdot \tilde{q} d \Gamma=\frac{1}{\alpha} \int_{\Omega} u^{*} \times b(Q, s) d \Omega .
$$

We denote for short

$$
b(Q, s)=\frac{1}{\alpha} u_{0}+\frac{1}{k_{0}} h(Q, s)=\frac{1}{\alpha} u_{0}+\frac{e^{\beta z}}{k_{0}} \times \tilde{h}\left(Q, s-\beta^{2} \alpha\right),
$$

A sequence of higher order fundamental solutions can be defined by the recurrence formula 
or by the formula

$$
\begin{aligned}
& u^{*(0)}=u^{*} \\
& \nabla^{2} u^{*(j+1)}=u^{*(j)}, \quad(j=0,1,2, \ldots), \\
& q^{*(j+1)}=\frac{\partial u^{*(j+1)}}{\partial n}
\end{aligned}
$$

$$
\begin{aligned}
& u^{*(j)}=\left(1 / \lambda^{j}\right) u^{*(0)}, \quad(j=0,1,2, \ldots) . \\
& q^{*(j)}=\left(1 / \lambda^{j}\right) q^{*(0)}
\end{aligned} .
$$

The higher order source functions are listed as follows:

$$
\begin{array}{ll}
h^{(0)}(Q, s)=h(Q, s) & b^{(0)}(Q, s)=(1 / \alpha) u_{0}(Q)+\left(1 / k_{0}\right) h^{(0)}(Q, s) \\
h^{(j)}(Q, s)=\nabla^{2} h^{(j-1)}(Q, s), & b^{(j)}=(1 / \alpha) u_{0}^{(j)}+\left(1 / k_{0}\right) h^{(j)} \\
& b_{n}^{(j)}=\frac{\partial b^{(j)}}{\partial n}=(1 / \alpha) \frac{\partial u_{0}^{(j)}}{\partial n}+\left(1 / k_{0}\right) \frac{\partial h^{(j)}}{\partial n}
\end{array} .
$$

Consider eqn. (18), we reform eqn. (15) into the following equation:

$$
u^{*}=(1 \backslash \lambda) \nabla^{2} u^{*}+(1 \backslash \lambda) \Delta(Y, Q)=(1 \backslash \lambda) u^{*(1)}+(1 \backslash \lambda) \Delta(Y, Q) .
$$

Using reciprocity scheme, the domain integral can be represented recursively by:

$$
\begin{aligned}
\int_{\Omega} u^{*(0)} \times b^{(0)} d \Omega & =\int_{\Gamma} q^{*} \times \sum_{j=0}^{\infty} \frac{1}{\lambda^{j+1}} b^{(j)} d \Gamma-\int_{\Gamma} u^{*} \times \sum_{j=0}^{\infty} \frac{1}{\lambda^{j+1}} b_{n}^{(j)} d \Gamma-c \sum_{j=0}^{\infty} \frac{1}{\lambda^{j+1}} b^{(j)}(Y) \\
& \approx \int_{\Gamma} q^{*} \times \sum_{j=0}^{M-1} \frac{1}{\lambda^{j+1}} b^{(j)} d \Gamma-\int_{\Gamma} u^{*} \times \sum_{j=0}^{M-1} \frac{1}{\lambda^{j+1}} b_{n}^{(j)} d \Gamma-c \sum_{j=0}^{M-1} \frac{1}{\lambda^{j+1}} b^{(j)}(Y)
\end{aligned}
$$

If the function $\mathrm{b}(Q, s)$ can be expressed by a polynomial function, $\mathrm{b}^{(j)}$ and $\mathrm{b}_{\mathrm{n}}{ }^{(j)}$ approach to zero with an increasing $\mathrm{j}$. Thus the infinite series of boundary integrals will become finite exactly. If the function $\mathrm{b}(Q, s)$ is more general, bearing in mind the convergence of the higher order fundamental solution that had been discussed in Reference [8], the infinite series can also be computed through several truncated terms.

\subsection{Transformation inversion}

After the analysis in Laplace Space, the numerical solution should be translated to the real space.

Firstly, the numerical solution in Laplace Space should be transferred into the time space by the Laplace inversion. The inversion formulation used in my implementation is Gaver function as follows:

$$
\begin{aligned}
& G_{0}^{(n)}=\frac{n \alpha}{t} \hat{f}(n \alpha / t), \quad(1 \leq n \leq 2 N) \\
& G_{k}^{(n)}=\left(1+\frac{n}{k}\right) G_{k-1}^{(n)}-\left(\frac{n}{k}\right) G_{k-1}^{(n+1)}, \quad(k \geq 1, n \geq k) . \\
& f_{k}(t)=G_{k}^{(k)}
\end{aligned}
$$

And the Wynn's rho algorithm [13] is used to improve the efficiency, which is given by the recursive algorithm 


$$
\rho_{-1}^{(n)}=0, \quad \rho_{0}^{(n)}=f_{n}(t), \quad \rho_{k}^{(n)}=\rho_{k-2}^{(n+1)}+\frac{k}{\rho_{k-1}^{(n+1)}-\rho_{k-1}^{(n)}}, \quad(n \geq 0, k \geq 1) .
$$

Then the approximation to $f(\mathrm{t})$ is obtained as

$$
f_{k}(t, N)=\rho_{N}^{(0)} .
$$

The integer $\mathrm{N}$ must be even. As $\mathrm{N}$ increases the accuracy of the approximant $f(\mathrm{t}, \mathrm{N})$ also increases. Using Wynn's rho algorithm, the relative error estimates

$$
\left|\frac{f(t)-f(t, N)}{f(t)}\right| \approx 10^{-0.8 N} .
$$

That is, number of significant digits in the approximant $f(\mathrm{t}, \mathrm{N})$ is about $\mathrm{N}$.

Finally, the solution should be transferred into the real space by employing the substitution in eqn. (7).

\section{Numerical examples}

To verify the efficiency of our method, solutions for two transient heat conduction problems in functionally graded materials, with different type of condition and with different geometries are presented in this section.

\subsection{Constant temperature on two faces of a FGM cube}

The problem of interest is shown in fig. 1. The cube initial temperature is zero. Then the top face of the cube at $z=0$ is maintained at a temperature of $T=100$, while the bottom face at $\mathrm{z}=1$ is zero. The remaining four faces are insulated (zero normal flux). The boundary conditions and initial conditions are

$$
\phi(x, y, 0 ; t)=0, \quad \phi(x, y, 1 ; t)=100, \quad \phi(x, y, z ; t=0)=0 .
$$

The thermal conductivity and the specific heat are taken to be

$$
\begin{aligned}
& k(x, y, z)=k_{0} e^{2 \beta z}=5 e^{3 z}, \\
& c(x, y, z)=c_{0} e^{2 \beta z}=1 e^{3 z} .
\end{aligned}
$$

The analytical solution for temperature is

$$
\phi(x, y, z ; t)=T \frac{1-e^{-2 \beta z}}{1-e^{-2 \beta L}}+\sum_{n=1}^{\infty} B_{n} \sin \frac{n \pi z}{L} e^{-\beta z} e^{-\left(n^{2} \pi^{2} / L^{2}+\beta^{2}\right) \alpha t},
$$

where $\mathrm{L}$ is the dimension of the cube (in the $\mathrm{z}$-direction). The boundary face method mesh has 150 elements and 576 nodes. The coefficient $\mathrm{N}$ in eqn. (26) is 6. Fig. 2 shows the time evolution of the temperature at points $\mathrm{z}=0.2,0.4,0.6$, 0.8 . Notice that the temperature variation matches the analytical solution. The solution of flux on the bottom is shown in fig. 3 .

In this example, the numerical solutions verify the efficiency of our method to solve the transient heat conductivity for FGM. 


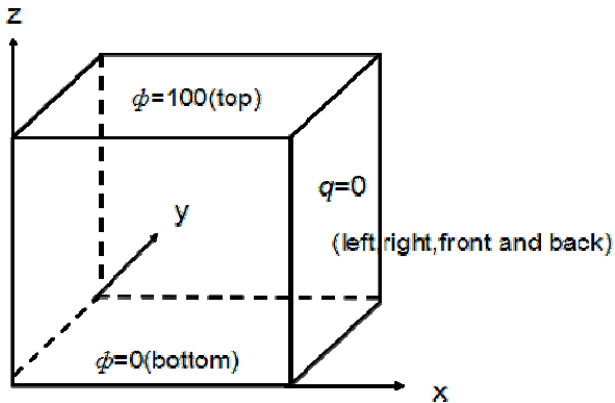

Figure 1: Geometry and boundary conditions of the FGM cube problem.

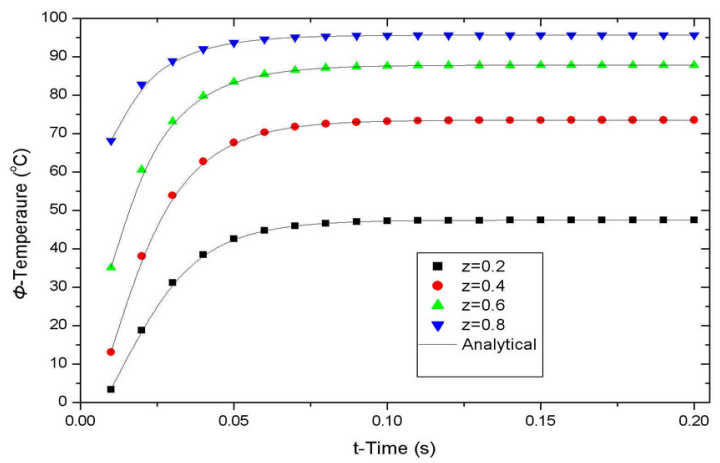

Figure 2: Temperature variation history on several points along the z-coordinate.

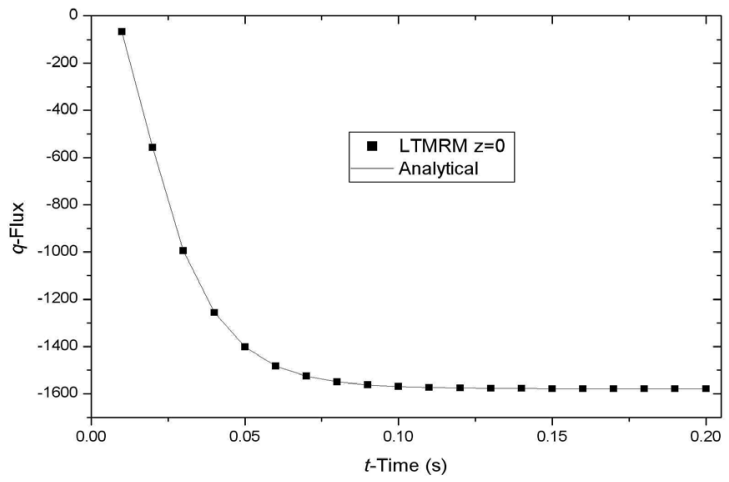

Figure 3: Flux variation history on the bottom face. 


\subsection{Quadratic heat source density in a FGM cube}

In this example we analyze a cube with a heat generation. The cube initial temperature is zero. Then the top surface and bottom surface of the cube at $\mathrm{z}=0$ and $\mathrm{z}=1$ is maintained at a temperature of $\mathrm{T}=0$ as shown in fig. 4 . The remaining four faces are insulated (zero normal flux). The length of the cube is $\mathrm{L}=1 \mathrm{~m}$. The thermal conductivity, the specific heat and the density are taken to be

$$
\begin{gathered}
k(x, y, z)=k_{0} e^{2 \beta z}=40 e^{20 z} \mathrm{~W} /(\mathrm{m} \cdot \mathrm{k}), \\
c(x, y, z)=c_{0} e^{2 \beta z}=0.5 e^{20 z} \times 10^{3} \mathrm{~J} /(\mathrm{kg} \cdot \mathrm{k}), \\
\rho=8 \times 10^{3} \mathrm{~kg} / \mathrm{m}^{3} .
\end{gathered}
$$

The heat generation is given by

$$
h(Q, t)=x^{2}+y .
$$

After using the substitution in eqn. (7), the heat density function in the diffusion equation becomes

$$
\tilde{h}(Q, t)=h(Q, t) \times e^{\beta z+\beta^{2} \alpha t}=\left(x^{2}+y\right) \times e^{10 z+0.001 t} .
$$

After Laplace transform, the heat density function is represented by

$$
h(Q, s)=\left(x^{2}+y\right) \times \frac{e^{\beta z}}{s-\beta^{2} \alpha}=\frac{\left(x^{2}+y\right) e^{10 z}}{s-0.001} .
$$

In this analysis, 150 boundary elements and 576 boundary nodes have been used. The coefficient $\mathrm{M}$ in eqn. (22) is 5 and $\mathrm{N}$ in eqn. (26) is 8 . Fig. 5 shows the time evolution of the temperature at different points. It is worth to observe that the temperature from the LTMRM matches the Abaqus solution within plotting accuracy. So the multiple reciprocity formulation is efficient to convert the domain integral.

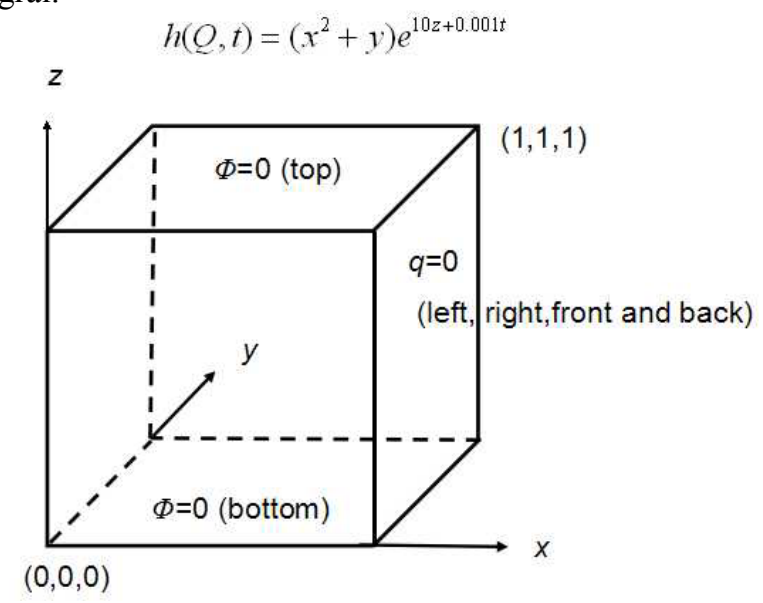

Figure 4: Geometry and boundary conditions of the FGM cube problem with the heat generation. 


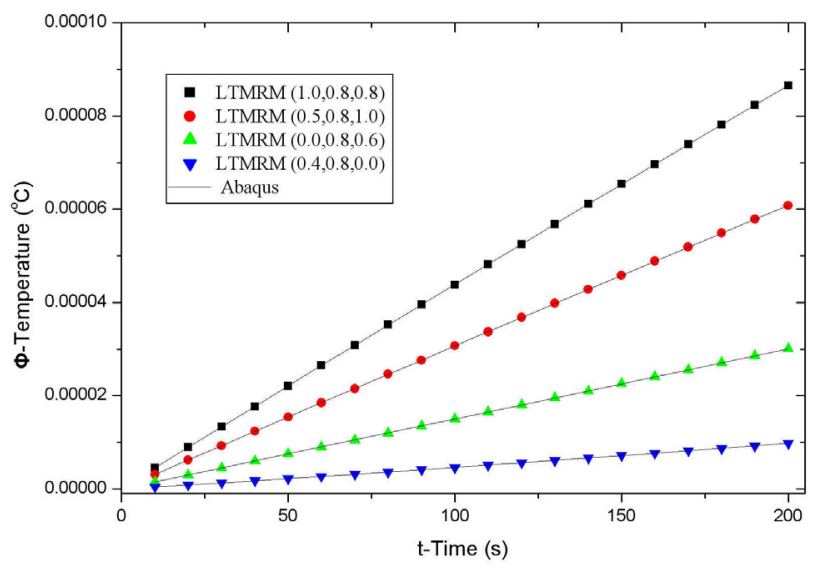

Figure 5: Temperature variation history on several points.

\section{Conclusion}

Three-Dimension transient heat conduction problems in functionally graded materials with heat generation are solved by the BFM which combines the LS and the MRM. The domain integrals are converted to boundary integrals successfully by the multiple reciprocity formulation. Numerical results illustrated the validity and accuracy of the proposed method.

In our method, with the quickly convergent higher order fundamental solutions, most of the domain integrals, which appear in the BIE of transient heat conduction problem in FGM, can be converted to several boundary integrals and non-integral terms. Furthermore, due to the simple form of the higher order fundamental solutions in our method, the implementation of our method is very simple and no additional matrix is required even for a large number of orders of the fundamental solutions. With the help of the Gaver-Wynn-rho formulation, we computed the inverse Laplace transformation accurately and efficiently in our implementation.

\section{Acknowledgements}

This work was supported in part by National 973 Project of China under grant number 2010CB328005, and in part by National Science Foundation of China under grant number 11172098 .

\section{References}

[1] Brebbia, C.A., Telles, J.C.F., Wrobel, L.C., Boundary Element Techniques, Springer-Verlag: Berlin and New York, pp. 11-13, 1984. 
[2] Rizzo, F.J., Shippy, D.J., A method of solution for certain problems of transient heat conduction. AIAA Journal, 8(11), pp. 2004-2009, 1970.

[3] Chang, Y., Kang, C., et al., The use of fundamental Green's functions for the solution of problems of heat conduction in anisotropic media. International Journal of Heat and Mass Transfer, 16(10), pp. 1905-1918, 1973.

[4] Tanaka, M., Matsumoto, T., et al., Dual reciprocity BEM based on timestepping scheme for the solution of transient heat conduction problems. International series on advances in boundary elements, pp. 299-308, 2002.

[5] Nowak, A.J., Neves, A.C., The multiple reciprocity boundary element method. Springer, pp. 45-67, 1994.

[6] Ochiai, Y., Kitayama, Y., Three-dimensional unsteady heat conduction analysis by triple-reciprocity boundary element method. Engineering analysis with boundary elements, 33(6), pp. 789-795, 2009.

[7] Gao, X., The radial integration method for evaluation of domain integrals. Engineering Analysis with Boundary Elements, 26, pp. 905-916, 2002.

[8] Guo, S., Zhang, J., et al., Three dimensional transient heat conduction analysis by Laplace transformation and multiple reciprocity boundary face method. Engineering analysis with boundary elements, 37(2), pp. 15-22, 2013.

[9] Sutradhar, A., Paulino, G.H., The simple boundary element method for transient heat conduction in functionally graded materials. Computer Methods in Applied Mechanics and Engineering, 193, pp. 4511-4539, 2004.

[10] Sladek, J., Sladek, V., Zhang, C.. Transient heat conduction analysis in functionally graded materials by the meshless local boundary integral equation method. Computational Materials Science, 28, pp. 494-504, 2003.

[11] Wang, H., Qin, Q.H., Kang, Y.L., A meshless model for transient heat conduction in functionally graded materials. computational mechanics, $\mathbf{3 8}$, pp. 5-60, 2006.

[12] Mirzaei, D., Dehghan, M., New implementation of MLBIE method for heat conduction analysis in functionally graded materials. Engineering Analysis with Boundary Elements, 36, pp. 511-519, 2012.

[13] Abate, J., Valkó, P., Multi-precision Laplace transform inversion. International Journal for Numerical Methods in Engineering, 60(5), pp. 979-993, 2004.

[14] Zhang, J., Qin, X., et al., A boundary face method for potential problems in three dimensions. International Journal for Numerical Methods in Engineering, 80(3), pp. 320-337, 2009.

[15] Qin X., Zhang, J., et al., A finite element implementation of the boundary face method for potential problems in three dimensions. Engineering analysis with boundary elements, 34, pp. 934-943, 2010. 